\title{
Epidemiology and Risk Factor of the Gallstone Disease in a Southern Tropical Country
}

\author{
Solonirina Davidà Rakotomena, Sedera Arimino*, Finaritra Casimir Fleur Prudence Rahantasoa, \\ Antso Hasina Raherinandrasana, Tianarivelo Rajaonarivony, Hery Nirina Rakoto Ratsimba
}

Faculty of Medecine, Antananarivo, Madagascar

Email address:

sederaarimino02@gmail.com (S. Arimino)

${ }^{*}$ Corresponding author

\section{To cite this article:}

Solonirina Davidà Rakotomena, Sedera Arimino, Finaritra Casimir Fleur Prudence Rahantasoa, Antso Hasina Raherinandrasana, Tianarivelo Rajaonarivony, Hery Nirina Rakoto Ratsimba. Epidemiology and Risk Factor of the Gallstone Disease in a Southern Tropical Country. European Journal of Clinical and Biomedical Sciences. Vol. 5, No. 6, 2019, pp. 73-78. doi: 10.11648/j.ejcbs.20190506.11

Received: November 12, 2019; Accepted: December 4, 2019; Published: December 13, 2019

\begin{abstract}
Despite the tendency to westernize our way of life, the Malagasy culture keeps its peculiarities. We report the epidemiological features of patients with cholelithiasis in our population in order to raise hypothesis about their risk factors. This is a retrospective descriptive multicenter and analytical case-control study of patients with cholelithiasis as "cases" and non-carriers of cholelithiasis as "controls". The odds ratios (ORs) were calculated with a $95 \%$ confidence interval to verify the correlation between food consumption and the occurrence of cholelithiasis. 51 patients were respectively enrolled for both groups. Consumption of animal products and legumes, such as offal (OR 95\% CI 3.23-1.36-7.67), chicken (OR 95\% CI 8.85 [3.02-25.9]), peas (OR 95\% CI 1.13 [0.43 - 2.95]) and Bambara peas (OR 95\% CI 1.31 [0.57 - 3.01 ]) would expose the risk of occurrence of cholelithiasis. In contrast, whole milk (OR 95\% CI 1.27 [0.58 - 2.77]), sweet potato (OR 95\% CI 0.72 [0.23 2.25]) and cassava (OR 95\% CI 0.83 [0.36 - 1.92]) would be a protective factor. To conclude, the diversification of our fruits and vegetables could be an asset to reduce the frequency of occurrence of cholelithiasis, giving priority to their consumption.
\end{abstract}

Keywords: Epidemiology, Gallstones, Madagascar, Risk Factor

\section{Introduction}

Gallstone is a common medical and surgical problem in both developed and non-developed countries [1, 2]. Gallstone is a potentially serious pathology by its numerous complications, but especially, studies had shown the association between the carcinoma of gall bladder and the gallstones [3, 4]. This pathology deserves a particular attention because of their increasing frequency, which varies from country to country [5]. The development of cholelithiasis is multifactorial. It has been well documented that the presence of gallstones increases with age and that predisposing risk factors for gallstone formation include obesity, diabetes mellitus, estrogen and pregnancy, hemolytic diseases and cirrhosis [6]. Furthermore, diet can be a modifiable risk factor to prevent gallstone disease [7-9]. And in our country, despite the impact of globalization on dietary habits and lifestyle, the Malagasy culture keeps its features.
The aim of this study is to report the features of the epidemiological profile of patients with cholelithiasis seen in our university hospitals and to make assumptions about the risk factors of this pathology.

\section{Materials and Methods}

\subsection{Study Design and Population}

This is a retrospective descriptive and analytic observational study in the three hospitals centers of Antananarivo, two of which were provided with surgical services: the Joseph Ravoahangy Andrianavalona University Hospital Center, the Soavinandriana Hospital Center and the Center. University Hospital Joseph Raseta Befelatanana.

Sampling was performed with a case-control ratio of 1 and a single-factor match which is the presence of hyperbilirubinemia $(>10 \mathrm{mg} / \mathrm{L}$ or $>17 \mu \mathrm{mol} / \mathrm{L})$. For "cases", an exhaustive recruitment of all carriers of gallstone, 
symptomatic or not, confirmed by a medical imaging examination, between 2015 and 2017, was done. And for "controls", we had taken patients admitted during this same period for another pathology in whom the presence of cholelithiasis was eliminated by a medical imaging examination.

The data collection was performed with a pre-established questionnaire including demographic profiles (sex, age, weight, size), medical history, lifestyle habits (smoking, alcohol, brew, physical activities) and food consumption frequency.

\subsection{Ethics Statements}

This study had respected the rules of ethics respecting autonomy, beneficence and justice, with information and consent of each participant on the purpose of the study. The procedures followed were also in accordance with the ethical standards of the responsible committee on human experimentation (institutional or regional) and with the Helsinki Declaration of 1975, as revised in 2000.
The association was performed with a univariate analysis of odds ratio (OR) and relative risk (RR), and 95\% confidence interval not including the value 1 was considered statistically significant. Furthermore, p-value of less than 0.05 was considered statistically significant.

\section{Results}

\subsection{Baselines Characteristics}

51 "cases" of which 12 had hyperbilirubinemia (23.52\%), were selected with a mean age of 48.68 years (16 to 77 years) and a female predominance of 31 cases (sex ratio equal to 0.65). About the 51 "controls" of which 12 also had hyperbilirubinemia (23.52\%), the mean age was 35.80 years (16 to 72 years) with a sex ratio equal to 1.43 indicating the male predominance.

On another hand, overweight patients accounted for $58.82 \%$ of patients with stones (30 cases) and 17.65 of nonlithiasis patients $(9$ cases). Their characteristics are summarized in Table 1.

\subsection{Statistical Analysis}

Table 1. Distribution of cases and controls by demographic and clinical variables.

\begin{tabular}{|c|c|c|}
\hline Characteristics & With gallstone (Cases) $n=51(\%)$ & Without gallstone (Controls) $n=51(\%)$ \\
\hline \multicolumn{3}{|l|}{ Age (years) } \\
\hline$\leq 40$ & $17(33,33)$ & $36(70,59)$ \\
\hline $41-60$ & $24(47,06)$ & $9(17,65)$ \\
\hline$\geq 60$ & $10(19,61)$ & $6(11,76)$ \\
\hline \multicolumn{3}{|l|}{ Gender } \\
\hline Feminine & $31(60,78)$ & $21(41,18)$ \\
\hline Masculine & $20(39,21)$ & $30(58,82)$ \\
\hline Diabete & $2(3,92)$ & $0(0)$ \\
\hline Sickle cells disease Acute pancreatitis Viral hepatitis B & $1(1,96) 3(5,88) 2(3,92)$ & $0(0) 0(0) 0(0)$ \\
\hline Family history of gallstone & $2(3,92)$ & $0(0)$ \\
\hline \multicolumn{3}{|l|}{ Body Mass Index $\left(\mathrm{kg} / \mathrm{m}^{2}\right)$} \\
\hline$<25$ & $21(41,18)$ & $42(82,35)$ \\
\hline $25-32$ & $28(54,90)$ & $9(17,65)$ \\
\hline$>32$ & $2(3,92)$ & $0(0)$ \\
\hline
\end{tabular}

\subsection{Analytic Study}

Women would be more exposed to gallstones than men (OR, 2.21; 95\% CI, 1.003 - 4.88).

Among the 31 women with gallstone, multiparity was noted in 19 patients $(61.29 \%, \mathrm{n}=31)$ versus six women in "controls" (28.57\%, $\mathrm{n}=21)$. About the use of hormonal contraception, eight "case" women used them $(25.81 \%, \mathrm{n}=$ $31)$, compared to six "control" patients $(28.57 \%, \mathrm{n}=21(\mathrm{OR}$, 0.95 ; $95 \%$ CI, 0.25 - 3.42). Then, hormonal contraception would be a protective factor for cholelithiasis (Table 2).

Table 2. Distribution of patients according to their gynecology and obstetric histories.

\begin{tabular}{llll}
\hline & With gallstone (Cases) $\mathbf{n}=\mathbf{5 1}(\mathbf{\%})$ & Without gallstone (Controls) $\mathbf{n}=\mathbf{5 1}(\mathbf{\%})$ & Odds ratio 95\% CI \\
\hline PARITY & & & 1 \\
Nulliparous & $2(6,45)$ & $5(23,82)$ & $2,5[0,39-16,05]$ \\
Pauciparous & $10(32,26)$ & $10(47,62)$ & $7,9[1,2-51]$ \\
Multiparous & $19(61,29)$ & $6(28,57)$ & 1 \\
CONTRACEPTIVE METHOD & & $15(71,43)$ & $0,95[0,25-3,42]$ \\
None & $19(61,29)$ & $6(28,57)$ & $\mathrm{U}^{*}$ \\
Hormonal & $8(25,81)$ & $0(0)$ & \\
Others & $4(12,90)$ & & \\
\hline
\end{tabular}

*U: unanalysed

Table 3 shows the association between tobacco, alcohol and decoction consummation and gallstone formation. Active 
smoking and chronic ethyl were found to increase the risk of gallstone formation by nearly two times (OR, 1.84; 95\% CI, 0.69 - 4.92; OR, 1.93; 95\% CI, 0.69 - 5.39). And a regular consumption of decoction seems to have less effect on it (OR, 1.17; 95\% CI, 0.39 - 3.51).

Table 3. Association between the occurrence of cholelithiasis and toxic habits.

\begin{tabular}{|c|c|c|c|}
\hline Toxic habits & With gallstone (Cases) $n=51(\%)$ & Without gallstone (Controls) $n=51(\%)$ & Odds ratio $95 \%$ CI \\
\hline \multicolumn{4}{|l|}{ Alcohol } \\
\hline Yes & $12(23,53)$ & $7(13,72)$ & $1,93[0,69-5,39]$ \\
\hline No & $39(76,47)$ & $44(86,27)$ & \\
\hline \multicolumn{4}{|l|}{ Decoction } \\
\hline Yes & $8(15,69)$ & $7(13,73)$ & $1,17[0,39-3,51]$ \\
\hline No & $43(84,31)$ & $44(86,27)$ & \\
\hline \multicolumn{4}{|l|}{ Tobacco } \\
\hline No & $38(74,51)$ & $43(84,31)$ & \\
\hline
\end{tabular}

The effects of dietary habits are shown in the table 4 . Consumption of meat and sausages is a risk factor of gallstone with a significant association (OR, [2.18-8.85]). Whereas, regular consumption of dry vegetables may be a protective factor with a significant association (OR, 0.38; $95 \%$ CI, $0.17-0.85$ ). Whole milk would be a protective factor against gallstone and could reduce the risk of gallstone formation; while the consumption of yoghurt would be a risk factor (OR, 1.27; 95\% CI, 0.58 - 2.77). Consisting vegetable oils and animal fats such as soybean oil and peanut oil would be protective factors and their consumption would respectively reduce the risk. In contrast, mayonnaise and coconut increase the risk of gallstone formation by respectively 1.26 and 1.74 times.
About cereals and its derivatives such as "mofogasy", made with rice flour, wheat flour and corn, they would be protective factors for gallstone. But regular consumption of "koba", a typical Malagasy recipe made of mixture of rice flour and peanut, increases the risk of developing cholelithiasis by two fold. Among fruits and vegetables, there was no association between potato consumption and gallstones (OR, 1; 95\% CI, 0.41 - 2.44). While consumption of cassava and sweet potato would reduce the risk of developing gallstones (respectively: OR, 0.83; $95 \% \mathrm{CI}, 0.36-1.92$ and OR, 0.72; 95\% CI, $0.23-2.25$ ). Finally, consumers of stir-fried vegetables would be five times more exposed to the development of gallstones (OR, 4.89; 95\% IC, 1.5 - 16.03).

Table 4. Association between the occurrence of cholelithiasis and the foods consumption.

\begin{tabular}{|c|c|c|c|}
\hline Foods & With gallstone (Cases) $n=51(\%)$ & Without gallstone (Controls) $n=51(\%)$ & Odds ratio $95 \% \mathrm{CI}$ \\
\hline \multicolumn{4}{|c|}{ MEAT AND DELI MEAT } \\
\hline Offal & $24(47,06)$ & $11(21,57)$ & $3,23[1,36-7,67]$ \\
\hline Deli meat & $21(41,18)$ & $8(15,69)$ & $3,76[1,47-9,61]$ \\
\hline Chicken & $25(49,02)$ & $5(9,80)$ & $8,85[3,02-25,9]$ \\
\hline Smoked meat & $8(15,69)$ & $4(7,84)$ & $2,19[0,61-7,79]$ \\
\hline \multicolumn{4}{|l|}{ FISH } \\
\hline River fish & $32(62,74)$ & $21(41,18)$ & $2,41[1,09-5,334]$ \\
\hline Fresh beans & $27(52,94)$ & $30(58,82)$ & $0,79[0,36-1,71]$ \\
\hline ried beans & $21(41,18)$ & $33(64,70)$ & $0,38[0,17-0,85]$ \\
\hline Peas & $11(21,57)$ & $10(19,61)$ & $1,13[0,43-2,95]$ \\
\hline Fresh bambara nuts & $18(35,29)$ & $15(29,41)$ & $1,31[0,57-3,01]$ \\
\hline \multicolumn{4}{|c|}{ FRUITS AND VEGETABLES } \\
\hline Stir-fried vegetables & $15(29,41)$ & $4(7,84)$ & $4,89[1,5-16,03]$ \\
\hline Cassava leaves & $27(52,94)$ & $26(50,98)$ & $1,08[0,5-2,35]$ \\
\hline Potato & $38(74,51)$ & $38(74,51)$ & $1[0,41-2,44]$ \\
\hline Fried potato & $33(64,70)$ & $32(62,74)$ & $1,09[0,49-2,44]$ \\
\hline Taro & $8(15,69)$ & $6(11,76)$ & $1,39[0,45-4,37]$ \\
\hline Cassava & $15(29,41)$ & $17(33,33)$ & $0,83[0,36-1,92]$ \\
\hline \multicolumn{4}{|c|}{ CEREALS AND DERIVED } \\
\hline Corn & $21(41,18)$ & $26(50,98)$ & $0,67[0,31-1,47]$ \\
\hline Soybeans & $10(19,61)$ & $10(19,61)$ & $1[0,38-2,66]$ \\
\hline Wheat flour & $8(15,69)$ & $16(31,37)$ & $0,42[0,16-1,09]$ \\
\hline Mofogasy & $27(52,94)$ & $33(64,70)$ & $0,61[0,28-1,35]$ \\
\hline Koba & $10(19,61)$ & $6(11,76)$ & $1,83[0,61-5,48]$ \\
\hline \multicolumn{4}{|l|}{ DAIRY PRODUCTS } \\
\hline Whole milk & $16(31,37)$ & $23(45,10)$ & $0,55[0,25-1,26]$ \\
\hline Yoghurt & $28(54,90)$ & $25(49,10)$ & $1,27[0,58-2,77]$ \\
\hline \multicolumn{4}{|l|}{ NUTS } \\
\hline Coconut & $5(9,80)$ & $3(5,88)$ & $1,74[0,39-7,7]$ \\
\hline
\end{tabular}




\begin{tabular}{llll}
\hline Foods & With gallstone (Cases) $\mathbf{n}=\mathbf{5 1}(\mathbf{\%})$ & Without gallstone (Controls) $\mathbf{n}=\mathbf{5 1}$ (\%) & Odds ratio 95\% CI \\
\hline Peanuts & $16(31,37)$ & $24(47,06)$ & $0,51[0,23-1,14]$ \\
ADDED FATS & & & \\
Mayonnaise & $18(35,29)$ & $13(25,49)$ & $1,26[0,68-3,73]$ \\
Soya oil & $31(60,78)$ & $34(66,67)$ & $0,77[0,35-1,75]$ \\
\hline
\end{tabular}

\section{Discussions}

The prevalence of cholelithiasis varies widely by nations, with a clear difference between Westerners (10-20\%) and Easterners $(2.4 \%$ to $6 \%)$, as well as between developed and developing countries $[5,10]$. Despite the tendency to imitate the lifestyle and diet of developed countries, the prevalence of cholelithiasis in our population still remains quite low of $0.01 \%$ with an average of 2 to 3 cases per month. However, their frequency may be underestimated due to the small size of our study population. In addition, the average age of subjects with cholelithiasis is similar with 47.34 years in the United States and Europe [10], $46.9 \pm 3$ years in Africa [11, 12] and 48.68 years in our series. And it was noted an increase in the frequency of cholelithiasis with age that would be explained by the increase in biliary secretion and intestinal absorption with age, as well as the decrease in synthesis and hepatic secretion of bile salts associated with hypomotility of the gallbladder in the elderly [5].

Apart from age, the genus also influences the occurrence of lithogenesis because women, especially those who are pregnant or using hormone replacement therapy, are more exposed to lithogenesis because of their higher estrogen levels. Then, there is an oversaturation of the bile in cholesterol, a change in plasma cholesterol concentration including a decrease in low-density lipoprotein (LDL) with elevation of high-density lipoprotein (HDL), associated to vesicular hypomotility [5]. And in our series, women were 2.21 times more affected by cholelithiasis than men. Other than that, literature suggests that metabolic syndrome which consists of the combination of obesity, hyperglycemia, dyslipidemia and arterial hypertension, constitute also a risk factor for cholelithiasis, with a risk of 2.6 times higher [13]. In this way, Maclure had specified that a BMI $>32 \mathrm{~kg} / \mathrm{m}^{2}$ would increase the risk of cholelithiasis by 6 times compared to a BMI $<22 \mathrm{~kg} / \mathrm{m}^{2}$, and a BMI between 24 and $25 \mathrm{~kg} / \mathrm{m}^{2}$ would give a relative risk of 1.7 [14]. In our study, the role of weight and BMI in lithogenesis was verified because we found that overweight patients were 6.66 times more likely to develop cholelithiasis compared to those with normal.

Furthermore, toxic habits also have a strong say in lithogenesis. Studies showed a relative risk ranging from 1.3 to 2.15 of cholelithiasis in tobacco users $[15,16]$. Concerning alcohol consumption, its results on the effect on the formation of cholelithiasis are contradictory. Some authors have raised its protective effect, but others consider rather its deleterious effects on health in general proscribing any prevention of cholelithiasis by the consumption of alcohol $[14,17]$.

In our study, the combination of smoking and alcoholism was not a true risk factor for gallstone disease. This result could be explained by our population sample which was dominated by non-ethyl-smoking patients. However, a special feature of Malagasy population is the frequent use of traditional herbal medicine which is the subject of many studies. And according to our study, a frequent consumption of decoction could expose to 1.17 times more risk of cholelithiasis. This risk may be poorly estimated but a larger study population size may provide better accuracy.

Concerning the impact of dietary habits, it is well known that gallstones are more common in the Western countries because of their high calorie diet, rich in cholesterol, saturated fatty acids, carbohydrates, refined sugar, in protein and salts, but low in fiber [5]. Our results corroborate those in the literature concerning the increased risk of occurrence of cholelithiasis in high calorie foods.

And among our eating habits, we particularly raise the potential threat, evaluated at 3.24 times more, by the consumption of giblets which are frequently consumed by Malagasy people for their price more affordable compared to other meats. Finally, as our country is rich in varieties of food plants, the study of exposure to various food plants was relevant. Cassava, maize, legumes and sweet potatoes are the staple food for our rural population. And our study did not find any significant risk for their consumption. However, our results were able to point out that sweet potato consumption may be a protective factor in the occurrence of gallstone. Paradoxically, our study also noted that some foods high in saturated fatty acids such as soybean oil and groundnuts would be protective factors.

Apart from that, similarity was observed between foods considered as risk factors for cardiovascular diseases and those that are lithogenic, particularly with regard to these foods rich in fatty acids [18]. Amani pointed out that coconut and butter would increase the risk of developing cardiovascular diseases by 2.35 times [19]. In our study, the consumption of coconut, which is often taken as an additional ingredient in our main meals, would expose to 1.74 times more risk of cholelithiasis.

And especially milk, findings from the literature are controversial. In fact, according to Ansari-Moghaddam, the consumption of dairy products is unrelated to the occurrence of cholelithiasis (OR, 1; 95\% CI, 0.72 - 3.50) [20]. As for its impact on the cardiovascular pathologies, Amani specified that milk, rich in fatty acid, would increase by 2.35 times the risk of their occurrence [19]. And in our study, whole milk would be a protective factor against cholelithiasis (OR, 0.55; $95 \%$ CI, 0.25 - 1.26) while yoghurt consumption would be a risk factor for lithiasis formation $(\mathrm{OR}, 1.27$; 95\% CI, 0.58 2.77).

Also, health lifestyle would have a concomitant beneficial effect on the cardiovascular than biliary health. And given the current socio-economic context of our country, preventive 
measures would be recommended for the general population in order to reduce the frequency of these pathologies which management remains expensive. First, physic activity involves its pro-kinetic effect on cholecystokinin-dependent vesicle contraction. Then, it is advised to perform regular physical activity and to maintain ideal weight in order to prevent the formation of cholesterol stone [1]. Secondly, consumption of fruits and vegetables should also be preferred because, according to Ansari-Moghaddam, low consumption of fruit and vegetables would increase the risk of cholelithiasis by twice (OR, 2.51; 95\% CI, 1.29 - 4.88) [20]. And one study about the dose-response correlation indicated that gallstone risk was reduced by $3 \%$ and $4 \%$ for every 200 g per day increment in fruits and vegetables consumption [8]. A nonvegetarian diet and hypercholesterolemia may have an additive effect in increasing GSD risk in women. Apart from that, actually, study showed that vegetarian diet was associated with a decreased risk of symptomatic gallstone disease compared with nonvegetarian diet in women (HR, $0.52 ; 95 \% \mathrm{CI}, 0.28-0.96)$ but not in men. In women, nonvegetarians with hypercholesterolemia had 3.8 times the risk of GSD compared with vegetarians with normal cholesterol (HR, 3.81; 95\% CI, 1.61 - 9.01) [9].

Finally, our study was a preliminary study based on a small sample size that is not representative of the Madagascar population and on a non-exhaustive and nonquantitative list of our diet. However, it had indeed to raise hypotheses on the risk factors for the occurrence of cholelithiasis in our population.

\section{Conclusion}

The prevalence of cholelithiasis is underevaluated in Madagascar despite previous studies because of limited accessibility of the abdominal ultrasound, especially in remote areas of the country. However, it is still considered lower than that observed in Western countries. Madagascar is well known for its richness in tropical fruits and vegetables, which is an asset in the prevention of the occurrence of cholelithiasis. On another hand, the country is made up of many regions with highly diversified food culture and habits which would be a major asset to prevent gallstone. The imitation of the Western way of life has been growing in recent decades. Then, a more in-depth and large-scale study of the risk factors for this pathology would be relevant.

\section{References}

[1] European Association for the Study of the Liver (EASL). Recommandations de Pratique Clinique sur la prévention, le diagnostic et le traitement des calculs biliaires selon l'EASL. Journal of Hepatology. 2016; 65: 146-81.

[2] Sanlorenzo M, Caldera D, Randriamitantsoa S, Rakotondrajao J, Razanadranaivo F. La lithiase biliaire dans le Sud de Madagascar. Médecine d'Afrique Noire. 1993, 40 (10): $585-588$.
[3] Cariati A, Piromalli E, Cetta F. Gallbladder cancers: associated conditions, histological types, prognosis, and prevention. Eur J Gastroenterol Hepatol. 2014; 26: 562-569.

[4] Jain K, Mohapatra T, Das P, Misra MC, Gupta SD, Ghosh M, et al. Sequential occurrence of preneoplastic lesions and accumulation of loss of heterozygosity in patients with gallbladder stones suggest causal association with gallbladder cancer. Ann Surg. 2014; 260: 1073-1080.

[5] Payen J-L, Muscari F, Vibert E, Ernst O, Pelletier G. Lithiase biliaire. Presse Med. 2011; 40: 567-80.

[6] Schirmer BD, Winters KL, Edlich RF. Cholelithiasis and cholecystitis. J Long Term Eff Med Implants. 2005; 15 (3): 329-38.

[7] Di Ciaula A, Wang DQ, Portincasa P. An update on the pathogenesis of cholesterol gallstone disease. Curr Opin Gastroenterol 2018; 34: 71-80.

[8] Zhang J-W, Xiong J-P, Xu W-Y, Sang X-T, Huang H-C, Bian $\mathrm{J}$, et al. Fruits and vegetables consumption and the risk of gallstone diasease. A systematic review and meta-analysis. Medicine. 2019; 98: 28-37.

[9] Chang C-M, Chiu THT, Chang C-C, Lin M-N, Lin C-L. PlantBased Diet, Cholesterol, and Risk of Gallstone Disease: A Prospective Study. Nutrients. 2019; 11: 335 - 48.

[10] Delabrousse E, Di Martino V, Aubry S, Fein F, Sarliève P, Carbonnel $\mathrm{F}$, et al. The choledochal ring sign: a specific finding in acute biliary pancreatitis. Abd Imaging. 2008; 33; 3: $337-4$.

[11] Stinton LM, Myers RP, Shaffer EA. Epidemiology of gallstones. Gastroenterol Clin North Am. 2010; 39: 157-69.

[12] Brugère $C$, Slim $K$, Fritsch $S$. How to treat common bile duct stones? Ann Chir. 2005; 130: 175-7.

[13] Ko CW, Lee SP. Role of ERCP in gallstone, Epidemiology and natural history of common bile duct stones and prediction of disease. Gastroenterology. 2002; 56; 6: 165-9.

[14] Maclure KM, Hayes KC, Colditz GA, Stampfer MJ, Speizer FE, Willett WC. Weight, diet, and the risk of symptomatic gallstones in middle-aged women. N Engl J Med. 1989; 321: 563-69.

[15] Hussain I, Ahmed HA. Hepatobiliary Manifestations of Sickle Cell Anemia. Gastroenterol Res. 2010; 3; 1: 1-8.

[16] Etminan M, Delaney JA, Bressler B, Brophy JM. Oral contraceptives and the risk of gallbladder disease: a comparative safety study. CMAJ. 2011; 183: 899-904.

[17] Leitzmann MF, Tsai CJ, Stampfer MJ, Rimm EB, Colditz GA, Willett WC, et al. Alcohol consumption in relation to risk of cholecystectomy in women. Am J Clin Nutr. 2003; 78: 339347.

[18] Carvalho RB, Nobre RS, Guimarães MR, Teixeira SE, Silva AR. Risk factor associated with the development of metabolic syndrome in children and adolescents. Acta Paul Enferm. 2016; 29; 4: 439-45.

[19] Yamada M, Wong FL, Fujiwara S, Tatsukawa Y, Suzuki G. Smoking and alcohol habits as risk factors for benign digestive diseases in a Japanese population: the radiation effects research foundation adult health study. Digestion. 2005; 71: 231-7. 
[20] Ansari-Moghaddam A, Khorram A, Miri-Bonjar M, Mohammadi M, Ansari. The prevalence and risk factors of gallstone among adults in South-East of Iran: A populationbased study. J Health Sc. 2016; 8; 4: 60-6. 